\title{
Radio Jets in Young Stellar Objects with the SKA
}

\author{
Guillem Anglada* \\ Instituto de Astrofísica de Andalucía, CSIC, Spain \\ E-mail: guillemeiaa.es
}

\section{Luis F. Rodríguez}

Centro de Radioastronomía y Astrofísica, UNAM, México

E-mail: 1.rodriguez@crya. unam.mx

\section{Carlos Carrasco-González}

Centro de Radioastronomía y Astrofísica, UNAM, México

E-mail: c.carrasco@crya.unam.mx

Jets and outflows are ubiquitous in the process of formation of stars since accretion is intimately associated with outflow. Free-free radio continuum emission in the centimeter domain is associated with these jets. The emission is weak, and sensitive telescopes are required to detect it.

One of the key problems in the study of outflows is to determine how they are accelerated and collimated. Observations in the $\mathrm{cm}$ range are most useful to trace the base of the ionized jets, close to the young central object and its accretion disk, where optical or near-IR images are obscured by the high extinction present. Radio recombination lines in jets (in combination with proper motions) should provide their 3D kinematics at very small scale (near their origin). SKA will be crucial to perform this kind of observations.

Thermal jets are associated with both low and high mass protostars. The ionizing mechanism of these radio jets appears to be related to shocks in the associated outflows, as suggested by the observed correlation between the centimeter luminosity and the outflow momentum rate. From this correlation and that with the bolometric luminosity of the driving star it will be possible to discriminate with SKA between unresolved HII regions and jets, and to infer physical properties of the embedded objects.

Some jets associated with young stellar objects (YSOs) show indications of non-thermal emission (negative spectral indices) in part of their lobes. Linearly polarized synchrotron emission has been found in the jet of $\mathrm{HH} \mathrm{80-81,} \mathrm{allowing} \mathrm{us} \mathrm{to} \mathrm{measure} \mathrm{the} \mathrm{direction} \mathrm{and} \mathrm{intensity} \mathrm{of} \mathrm{the} \mathrm{jet} \mathrm{magnetic}$ field, a clue ingredient in determining the collimation and ejection mechanisms. As only a fraction of the emission is polarized, very sensitive observations such as those that will be feasible with SKA are required to perform these studies.

Jets are common in many kinds of astrophysical scenarios. Characterizing radio jets in YSOs, where thermal emission allows us to determine their physical conditions in a reliable way, would be also useful in understanding acceleration and collimation mechanisms in all kinds of astrophysical jets.

Advancing Astrophysics with the Square Kilometre Array

June 8-13, 2014

Giardini Naxos, Italy

\footnotetext{
* Speaker.
} 


\section{Introduction}

In the last years it has become clear that collimated outflows are present in young stars across all the stellar spectrum, from O-type protostars to brown dwarfs, suggesting that the disk-jet scenario is valid to describe the formation of stars of all masses.

Outflows associated with young stars frequently exhibit a central weak centimeter emission source (Anglada et al. 1992; Anglada 1995; Rodríguez \& Reipurth 1998). In the best studied cases, these sources are resolved angularly at the sub-arcsec scale and found to be elongated in the direction of the large-scale tracers of the outflow (e.g. Rodríguez et al. 1990; Rodríguez 1995, 1996; Anglada 1996), indicating that they trace the region, very close to the exciting star, where the outflow phenomenon originates. The centimeter flux density usually rises slowly with frequency (positive spectral index). Given their morphology and spectrum these sources are usually referred to as "thermal" radio jets. These radio jets have been found during all the stages of the star-formation process, from Class 0 protostars to stars associated with transitional disks, suggesting that outflow and accretion are intimately related (Rodríguez et al. 2014).
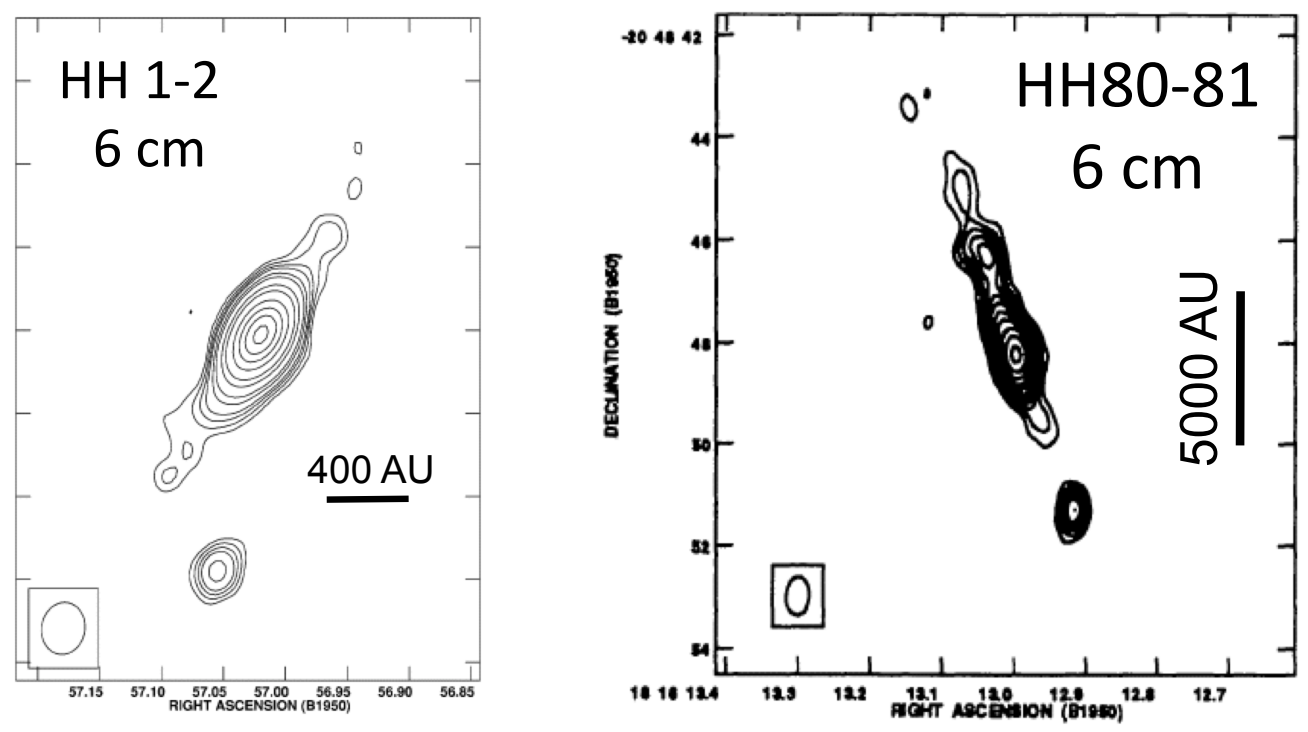

Figure 1: (Left) HH1-2 radio jet, associated with a low-mass object (Rodríguez et al. 2000). (Right) HH80-81 radio jet, associated with a high-mass object (Martí et al. 1993).

With the extraordinary sensitivity of the Jansky VLA, e-MERLIN, and the SKA it is expected that all nearby (a few kpc) young stellar objects (YSOs) known to be associated with outflows, both molecular and/or optical/infrared, will be detectable as centimeter sources (Anglada et al. 2015, in preparation). The topic of radio jets from young stars has been reviewed by Anglada (1996) and Rodríguez (1997; 2011).

\section{Properties of Radio Jets}

The study of jets associated with young stars at radio wavelengths is important in several aspects. Given the large obscuration present towards the very young stars, the detection of the 
radio jet provides so far the best way to obtain their accurate positions. These observations also provide information on the direction and collimation of the gas ejected by the young system in the last few years (see Table 1) that can be compared with the gas in the molecular outflows and optical/infrared $\mathrm{HH}$ jets, which traces the ejection over timescales several orders of magnitude larger. This comparison allows us to make evident the changes in the ejection direction, possibly resulting from precession or orbital motions in binary systems (Rodríguez et al. 2008; Masqué et al. 2012).

The radio jets from YSOs can be described using the free-free models of Reynolds (1986). In particular, for a jet of constant temperature, velocity and ionization fraction, these models predict that the flux density increases with frequency as $S_{v} \propto v^{\alpha}$, where $\alpha=1.3-0.7 / \varepsilon$, and $\varepsilon$ is the index of the power-law dependence of the jet width with distance to its origin. The case of $\varepsilon=1$ corresponds to a conical (constant opening angle) jet. The angular size of the major axis of the jet decreases with frequency as $\theta_{v} \propto v^{-0.7 / \varepsilon}=v^{\alpha-1.3}$. This behavior in flux density and size can be tested with multi-frequency observations.

With the Reynolds (1986) models it is also possible to determine from the observations the ionized mass loss rate, $\dot{M}_{i}$, in the jet and the radius at which ionization starts, $r_{0}$. The jet velocity, $V_{j}$, necessary to determine $\dot{M}_{i}$ can be estimated from proper motion measurements obtained from multi-epoch observations. In Table 1 we present the parameters of a few selected radio jets. These well-known jets have centimeter flux densities of $1 \mathrm{mJy}$ or more. However, most of the jets associated with low mass stars or even brown dwarfs present centimeter flux densities in the order of tens of $\mu \mathrm{Jy}$ (Rodríguez et al. 2015, in preparation) and their detection and study will require of the high sensitivity of SKA.

Table 1: Properties of Selected Angularly Resolved Radio Jets in YSOs

\begin{tabular}{|c|c|c|c|c|c|c|c|c|c|c|c|c|c|}
\hline Source & $\begin{array}{l}L_{\mathrm{bol}} \\
\left(L_{\odot}\right)\end{array}$ & $\begin{array}{c}M_{\star} \\
\left(M_{\odot}\right)\end{array}$ & $\begin{array}{c}d \\
(\mathrm{kpc})\end{array}$ & $\begin{array}{c}S_{V} \\
(\mathrm{mJy})\end{array}$ & $\alpha$ & $\begin{array}{c}\theta_{0} \\
(\mathrm{deg})\end{array}$ & $\begin{array}{l}\text { Size } \\
(\mathrm{AU})\end{array}$ & $\begin{array}{c}V_{j} \\
\left(\mathrm{~km} \mathrm{~s}^{-1}\right)\end{array}$ & $\begin{array}{l}t_{\mathrm{dyn}} \\
(\mathrm{yr})\end{array}$ & $\varepsilon$ & $\begin{array}{c}\dot{M}_{i} \\
\left(M_{\odot} \mathrm{yr}^{-1}\right)\end{array}$ & $\begin{array}{c}r_{0} \\
(\mathrm{AU})\end{array}$ & Refs. \\
\hline HH 1-2 VLA1 & 20 & $\sim 1$ & 0.4 & 1 & 0.3 & 19 & 200 & 270 & 2 & 0.7 & $1 \times 10^{-8}$ & $\leq 11$ & $1,2,3,4$ \\
\hline NGC 2071-IRS3 & $\sim 500$ & 4 & 0.4 & 3 & 0.6 & 40 & 200 & $400^{a}$ & 3 & 1 & $2 \times 10^{-7}$ & $\leq 18$ & $5,6,2,7$ \\
\hline Cep A HW2 & $1 \times 10^{4}$ & $4 \quad 15$ & 0.7 & 10 & 0.7 & 14 & 400 & 460 & 3 & 0.9 & $5 \times 10^{-7}$ & $\leq 60$ & $8,9,10,11,12$ \\
\hline HH 80-81 & $2 \times 10^{4}$ & $4 \quad 15$ & 1.7 & 5 & 0.2 & 34 & 1500 & 1000 & 7 & 0.6 & $1 \times 10^{-6}$ & $\leq 25$ & $13,14,15,16,17,18$ \\
\hline
\end{tabular}

${ }^{a}$ Assumed.

References: (1) Fischer et al. 2010; (2) Menten et al. 2007; (3) Rodríguez et al. 1990; (4) Rodríguez et al. 2000; (5) Butner et al. 1990; (6) Carrasco-González et al. 2012a; (7) Torrelles et al. 1998; (8) Hughes et al. 1995; (9) Patel et al. 2005; (10) Dzib et al. 2011; (11) Curiel et al. 2006; (12) Rodríguez et al. 1994; (13) Aspin \& Geballe 1992; (14) Fernández-López et al. 2011; (15) Rodríguez et al. 1980; (16) Martí et al. 1995; (17) Martí et al. 1993; (18) Carrasco-González et al. 2012b.

Sensitive, very high angular resolution observations at centimeter wavelengths can trace the base of the jets down to the injection radius, at scales of a few AUs, where the ionized jet is expected to begin. Exploration of this inner region will eventually shed new light on the jet acceleration and collimation mechanisms, helping to distinguish between different theoretical approaches such as the X-wind (Shu et al. 2000) and disk-wind (Königl \& Pudritz 2000) models. Shang et al. (2004) use the X-wind model to calculate the expected properties of the centimeter free-free emission of YSO jets. A comparison of detailed modeling results and high quality observations can provide an 
accurate description of the jet physical parameters.

Additionally, we note that a good knowledge of the jet properties is indispensable for grain growth studies in protoplanetary disks to separate the dust emission of the disk from the free-free emission of the jet. It is known that even transitional disks present free-free emission in their central regions (Rodríguez et al. 2014).

\section{Bolometric Luminosity, Outflow, and Radio Continuum Correlations}

Photoionization does not appear to be the ionizing mechanism of radio jets, since in the sources associated with low-luminosity objects, the number of UV photons from the star is clearly insufficient to produce the ionization required to explain the observed radio continuum emission (e.g., Rodríguez et al. 1989; Anglada 1995). The radio luminosity of radio jets $\left(S_{v} d^{2}\right)$ is correlated with the bolometric luminosity of the source, $L_{\mathrm{bol}}$, and with the momentum rate in the molecular outflow, $\dot{P}$ (Anglada 1995, 1996; see Fig. 2):

$$
\begin{gathered}
\left(\frac{S_{v} d^{2}}{\mathrm{mJy} \mathrm{kpc}^{2}}\right)=0.008\left(\frac{L_{\mathrm{bol}}}{L_{\odot}}\right)^{0.6} \\
\left(\frac{S_{v} d^{2}}{\mathrm{mJy} \mathrm{kpc}^{2}}\right)=190\left(\frac{\dot{P}}{M_{\odot} \mathrm{yr}^{-1} \mathrm{~km} \mathrm{~s}^{-1}}\right)^{0.9} .
\end{gathered}
$$
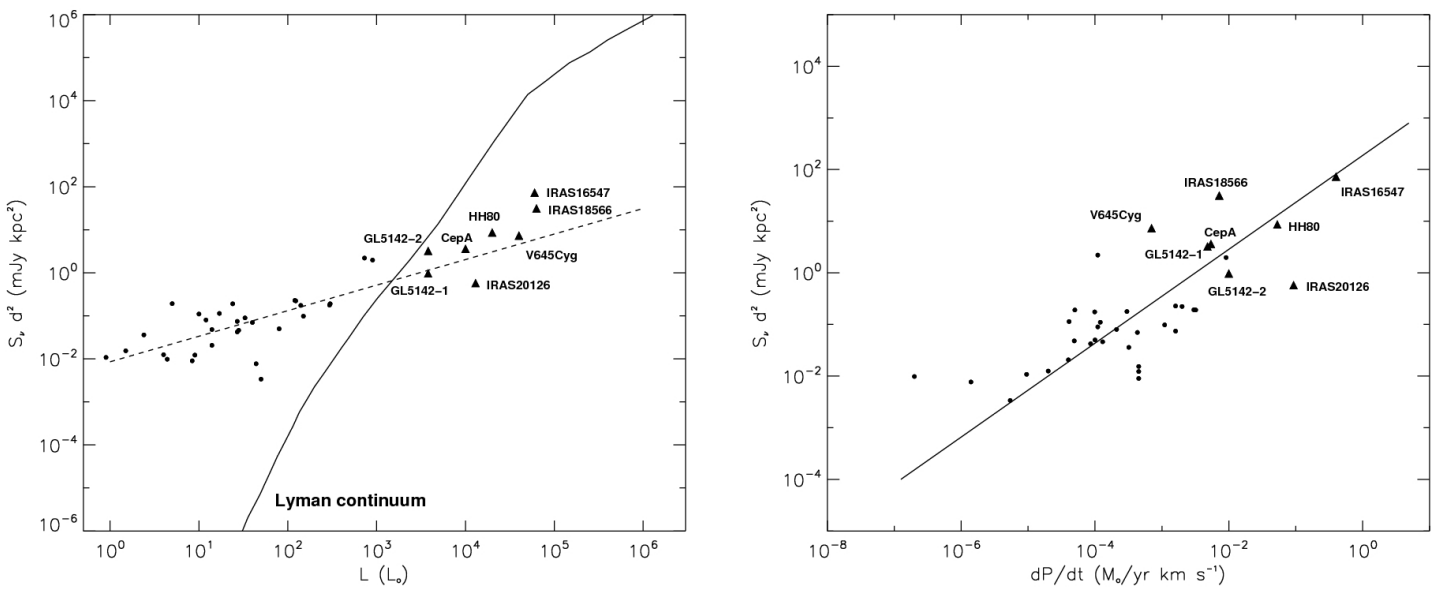

Figure 2: (Left) Radio luminosity versus bolometric luminosity correlation for young stellar objects (dashed line). The solid line (Lyman continuum) represents the radio luminosity expected from photoionization. (Right) Radio luminosity versus outflow momentum rate (solid line). The high luminosity objects are labeled in the figures.

These correlations include young stellar objects with luminosities spanning from 1 to $10^{5}$ $L_{\odot}$. In particular, these diagrams can be used to discriminate between the thermal radio jets (that should follow these correlations) and HII regions (that should fall close to the Lyman continuum line). Also, detection of radio recombination lines (RRLs, see Sect. 4) in jets could be useful to distinguish between jets and HII regions, since lines are expected to be broader in jets, as noted by Hoare et al. (2007) (see their Fig. 6). 


\section{Radio Recombination Lines}

Radio jets are expected to show RRLs as part of the emission processes of the plasma. RRLs in jets (in combination with proper motions) should provide their 3D kinematics at very small scale (near their origin).

Assuming local thermodynamic equilibrium (LTE) for line and continuum and a standard biconical jet with constant velocity and ionized fraction, the expected line to continuum flux density ratio in the centimeter wavelength regime is given by (Rodríguez et al. 2015, in preparation):

$$
\frac{S_{L}}{S_{C}}=0.19\left(\frac{v_{L}}{\mathrm{GHz}}\right)^{1.1}\left(\frac{T}{10^{4} \mathrm{~K}}\right)^{-1.1}\left(\frac{\Delta V}{\mathrm{~km} \mathrm{~s}^{-1}}\right)^{-1}\left(1+Y^{+}\right)^{-1} .
$$

In the above equation, $v_{L}$ is the frequency of the line, $\Delta V$ the full width at half maximum of the line, and $Y^{+}$is the ionized helium to ionized hydrogen ratio. Since the lines from a jet are expected to be wide, $\Delta V \simeq 100 \mathrm{~km} \mathrm{~s}^{-1}$, their detectability will be challenging. For example, at $10 \mathrm{GHz}$ the line-to-continuum ratio is expected to be $\sim 0.02$. Thus, for a jet with a continuum flux density of $1 \mathrm{mJy}$, the peak line flux density will be only $\sim 20 \mu \mathrm{Jy}$. So far, there are no detections of RRLs in jets at the expected LTE level. Jiménez-Serra et al. (2011) report the detection, at millimeter wavelengths, of broad recombination maser lines toward the jet in Cepheus A HW2 with flux densities about 5 times larger than those expected for LTE. Additional observations are needed to understand the nature of RRLs from jets and start using them as tools to study the outflow kinematics.

\section{Non-thermal Emission}

As discussed above, jets from YSOs have been long studied at radio wavelengths through their thermal free-free emission which traces the base of the ionized jet, and shows a characteristic positive spectral index. However, in the last two decades, negative spectral indices have been found in some regions of YSO jets (e.g., Rodríguez et al. 1989, 2005; Curiel et al. 1993; Martí et al. 1993; Garay et al. 1996; Wilner et al. 1999; see Fig. 3 left). This negative spectral-index emission is usually found in strong radio knots, that usually appear in pairs, sometimes found moving away from the central protostar at velocities of several hundreds of kilometers per second. Because of these characteristics, it has been proposed that these knots would be tracing strong shocks of the jet against dense material in the surrounding molecular cloud. Their negative spectral indices have been interpreted as indicating non-thermal synchrotron emission from a small population of relativistic particles that would be accelerated in the ensuing strong shocks.

This scenario has been confirmed only very recently, with detection of linearly polarized emission from the HH 80-81 jet (Carrasco-González et al. 2010; see Fig. 3 right). This result provided for the first time conclusive evidence for the presence of synchrotron emission in a YSO jet making it possible the direct measure and study of the properties of the magnetic field strength and morphology. Measuring linear polarization in YSO jets is difficult because it is only a fraction of the total emission. Ultrasensitive radio interferometers, such as the SKA, will allow us to detect and image the magnetic field in a large sample of YSO jets. In combination with the physical parameters (density, temperature, velocity) derived from observations of the thermal component, 

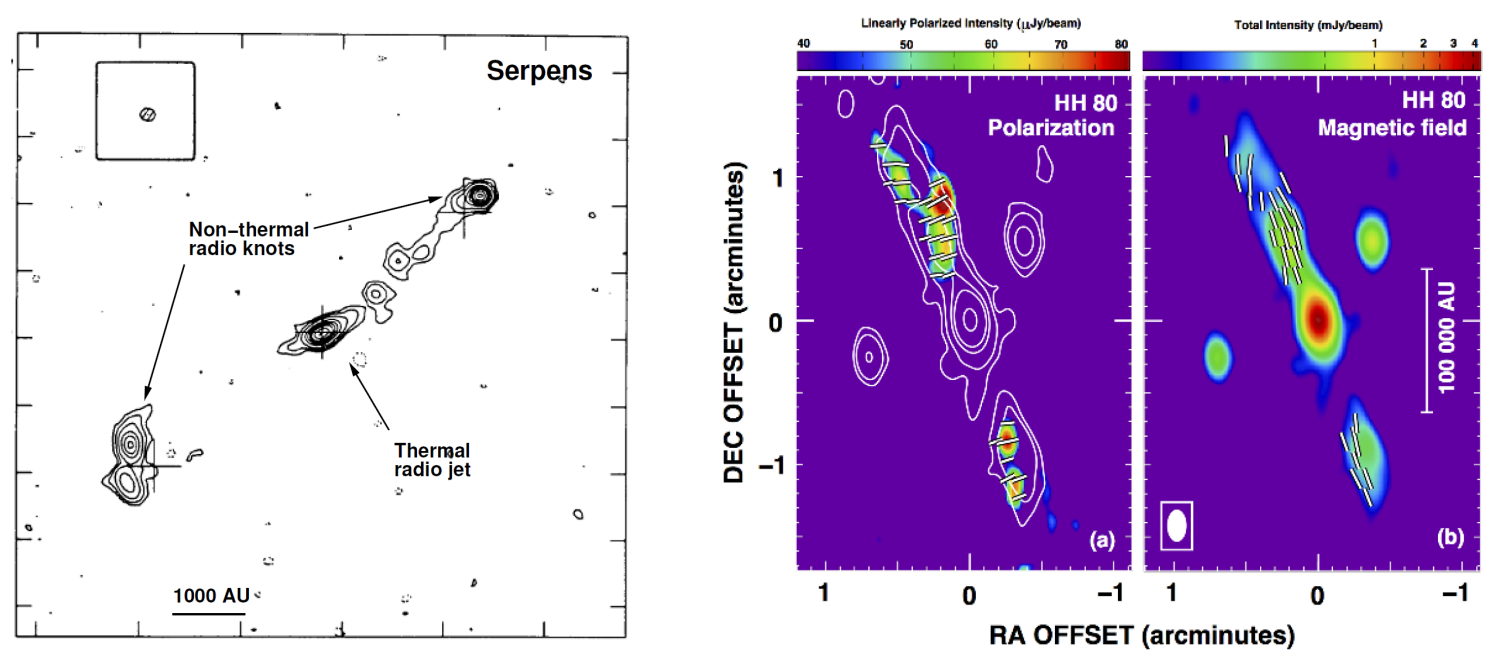

Figure 3: (Left) VLA image at $3.6 \mathrm{~cm}$ of the radio jet in Serpens, one of the first YSO jets where synchrotron emission was proposed to arise as a result of strong shocks with the ambient medium. The central source shows a positive spectral index at radio wavelengths, consistent with free-free emission, while the two bright knots at the end of the lobes show negative spectral indices indicative of thermal emission (adapted from Curiel et al. 1993). (Right) VLA images at $6 \mathrm{~cm}$ of the HH 80-81 jet showing the first detection of linearly polarized synchrotron emission in a YSO jet. Panel (a) shows in color scale the polarized emission and the polarization direction as white bars. Panel (b) shows the direction of the magnetic field as white bars. The total continuum intensity is shown in contours (a) and in color scale (b) (from Carrasco-González et al. 2010).

the measurement of the magnetic field from the non-thermal component will help in understanding YSO jet acceleration and collimation mechanisms, that appear to be similar for all kinds of astrophysical objects.

\section{Expectations for SKA Observations of Radio Jets}

The study of radio jets will greatly benefit from the higher frequency bands of SKA. In particular, given the rising spectrum and compact size of the radio jets, we anticipate that most observations of these sources will be carried out in band 5. SKA will allow us to survey the southern hemisphere for radio jets associated with young stars across the mass spectrum, including protobrown dwarfs. At a distance of $500 \mathrm{pc}$, a radio jet from a proto-brown dwarf is estimated to have a flux density of $12 \mu \mathrm{Jy}$ at $10 \mathrm{GHz}$ (Palau et al. 2015, in preparation). The SKA1-MID will detect this weak emission at the $10-\sigma$ level in only 20 minutes of on-source integration time. Detection of the jets from the most massive protostars (with expected radio luminosities of $10-100 \mathrm{mJy} \mathrm{kpc}{ }^{2}$, see Fig. 1) will in principle be feasible with at least a similar signal-to-noise ratio across the whole Galaxy. In the "early science" phase of SKA1 the detection of jets from brown dwarfs will reach $300 \mathrm{pc}$ and in the case of massive protostars, it will cover most of the Galaxy.

Observations at different frequencies across band 5 (providing angular resolutions in the 30 to 80 mas range) will determine the variations of the physical parameters along the jet axis and to image the region around the injection radius of the ionized gas in the jet. However, the high angular resolution study of radio jets with SKA1-MID only will be possible for jets of relatively 
high flux density (a few tenths of mJy). For weaker jets the full SKA (SKA2) will be required, since the uniform weighting SKA1 sensitivity is expected to be several times worst than for natural weighting (unless the antenna configuration is changed to favor longer baselines). For example, mapping the jet of a proto-brown dwarf is expected to take about 1.5 hours with SKA2, while it would take several hundreds of hours with SKA1.

A search for linear polarization using SKA1-MID will be feasible in the jets with relatively bright non-thermal knots. These knots have characteristic flux densities of about $100 \mu \mathrm{Jy}$. Assuming a linear polarization degree of $10 \%$, the detection of the Stokes parameters at a signal-to-noise ratio of 10, will require on-source integration times of order 30 minutes (about 2 hours in the "early science" phase of SKA1). With the full SKA (SKA2) it will be possible to search for linear polarization, and even to perform a high angular resolution mapping, in a much more extended sample.

For radio jets with continuum flux densities of order $3 \mathrm{mJy}$, a peak RRL flux density of 75 $\mu \mathrm{Jy}$ is expected (assuming a line width of about $100 \mathrm{~km} \mathrm{~s}^{-1}$, see formula 4.1). Adopting a velocity resolution comparable to the line width, a 6- $\sigma$ detection will be achieved with an on-source integration time of 1 hour with SKA1-MID ( $4 \mathrm{~h}$ in the "early science" phase of SKA1). If the emission is resolved spatially or we want better velocity resolution, the on-source time will increase accordingly. On the other hand, many recombination lines will be available in the SKA bands and it will be possible to stack them and improve the detectability (there are 35 RRLs in band 5, improving signal-to-noise ratio by a factor of $\sim 6$ ). This type of observations will reach its full potential with the full SKA (SKA2), when it will be possible to map the radial velocity distribution of the jet and in combination with proper motions obtain the 3D kinematics.

Finally, the high sensitivity and angular resolution of SKA will make it possible to study the equatorial winds, probably driven by radiation pressure acting on the gas on the surface layers of the disk, that have been observed in a number of massive young stars, and to distinguish this kind of sources from jets, as has been done in S140 IRS1 with MERLIN by Hoare (2006).

\section{Acknowledgments}

GA acknowledges support from MICINN (Spain) grant AYA2011-30228-C03 (co-funded with FEDER funds) and partial support from Junta de Andalucía (TIC-126). LFR and CC-G acknowledge support from DGAPA, UNAM and CONACyT (Mexico).

\section{REFERENCES}

Anglada, G. 1995, RevMexAA Conf. Ser., 1, 67

Anglada, G. 1996, Radio Emission from the Stars and the Sun, ASP Conf. Ser., 93, 3

Anglada, G., Rodríguez, L. F., Cantó, J., Estalella, R., \& Torrelles, J. M. 1992, ApJ, 395, 494

Aspin, C., \& Geballe, T. R. 1992, A\&A, 266, 219

Butner, H. M., Evans, N. J., II, Harvey, P. M., et al. 1990, ApJ, 364, 164

Carrasco-González, C., Galván-Madrid, R., Anglada, G., et al. 2012b, ApJL, 752, L29

Carrasco-González, C., Osorio, M., Anglada, G., et al. 2012a, ApJ, 746, 71

Carrasco-González, C., Rodríguez, L. F., Anglada, G., et al. 2010, Science, 330, 1209 
Curiel, S., Ho, P. T. P., Patel, N. A., et al. 2006, ApJ, 638, 878

Curiel, S., Rodríguez, L. F., Moran, J. M., \& Cantó, J. 1993, ApJ, 415, 191

Dzib, S., Loinard, L., Rodríguez, L. F., Mioduszewski, A. J., \& Torres, R. M. 2011, ApJ, 733, 71

Fernández-López, M., Girart, J. M., Curiel, S., et al. 2011, AJ, 142, 97

Fischer, W. J., Megeath, S. T., Ali, B., et al. 2010, A\&A, 518, L122

Garay, G., Ramírez, S., Rodríguez, L. F., Curiel, S., \& Torrelles, J. M. 1996, ApJ, 459, 193

Hoare, M. G. 2006, ApJ, 649, 856

Hoare, M. G., Kurtz, S. E., Lizano, S., Keto, E. Hofner, P. 2007, Protostars and Planets V, 181

Hughes, V. A., Cohen, R. J., \& Garrington, S. 1995, MNRAS, 272, 469

Jiménez-Serra, I., Martín-Pintado, J., Báez-Rubio, A., Patel, N., \& Thum, C. 2011, ApJL, 732, L27

Königl, A., \& Pudritz, R. E. 2000, Protostars and Planets IV, 759

Martí, J., Rodríguez, L. F., \& Reipurth, B. 1993, ApJ, 416, 208

Martí, J., Rodríguez, L. F., \& Reipurth, B. 1995, ApJ, 449, 184

Masqué, J. M., Girart, J. M., Estalella, R., Rodríguez, L. F., \& Beltrán, M. T. 2012, ApJL, 758, L10

Menten, K. M., Reid, M. J., Forbrich, J., \& Brunthaler, A. 2007, A\&A, 474, 515

Patel, N. A., Curiel, S., Sridharan, T. K., et al. 2005, Nature, 437, 109

Reynolds, S. P. 1986, ApJ, 304, 713

Rodríguez, L. F. 1995, RevMexAA Conf. Ser., 1, 1

Rodríguez, L. F. 1996, RevMexAA Conf. Ser., 4, 7

Rodríguez, L. F. 1997, IAU Symposium, 182, 83

Rodríguez, L. F. 2011, IAU Symposium, 275, 367

Rodríguez, L. F., Curiel, S., Moran, J. M., et al. 1989, ApJL, 346, L85

Rodríguez, L. F., Delgado-Arellano, V. G., Gómez, Y., et al. 2000, AJ, 119, 882

Rodríguez, L. F., Garay, G., Brooks, K. J., \& Mardones, D. 2005, ApJ, 626, 953

Rodríguez, L. F., Garay, G., Curiel, S., et al. 1994, ApJL, 430, L65

Rodríguez, L. F., Ho, P. T. P., Torrelles, J. M., Curiel, S., \& Cantó, J. 1990, ApJ, 352, 645

Rodríguez, L. F., Moran, J. M., Ho, P. T. P., \& Gottlieb, E. W. 1980, ApJ, 235, 845

Rodríguez, L. F., Myers, P. C., Cruz-González, I., \& Terebey, S. 1989, ApJ, 347, 461

Rodríguez, L. F., Moran, J. M., Franco-Hernández, R., et al. 2008, AJ, 135, 2370

Rodríguez, L. F., \& Reipurth, B. 1998, RevMexAA, 34, 13

Rodríguez, L. F., Zapata, L. A., Dzib, S. A., et al. 2014, ApJL, 793, L21

Shang, H., Lizano, S., Glassgold, A., \& Shu, F. 2004, ApJL, 612, L69

Shu, F. H., Najita, J. R., Shang, H., \& Li, Z.-Y. 2000, Protostars and Planets IV, 789

Torrelles, J. M., Gómez, J. F., Rodríguez, L. F., et al. 1998, ApJ, 505, 756

Wilner, D. J., Reid, M. J., \& Menten, K. M. 1999, ApJ, 513, 775 